\title{
O PERFIL DAS PUÉRPERAS ADOLESCENTES ATENDIDAS EM UMA MATERNIDADE DE REFERÊNCIA DE FORTALEZA-CEARÁ
}

\author{
The profile of the puerperal adolescents treated in a referenced maternity in Fortaleza- \\ Ceará
Perfil de las madres adolescentes atendidas en una maternidad de referencia en Fortaleza- Ceará

Náira de Oliveira Caminha'

Deise Maria do Nascimento Sousa ${ }^{4}$
Camila Chaves da Costa ${ }^{2}$

Lydia Vieira Freitas ${ }^{5}$
Raquel Ferreira Gomes Brasil ${ }^{3}$

Ana Kelve de Castro Damasceno ${ }^{6}$

\section{RESUMO}

Objetivou-se caracterizar o perfil das puérperas adolescentes de uma maternidade de For taleza-CE. Estudo descritivo, transversal e quantitativo, realizado no Alojamento Conjunto da Maternidade Escola Assis Chateaubriand, no período de março a julho de 2009, com 200 adolescentes. Os dados foram obtidos por meio de entrevistas. A maioria apresentava idade entre 15 e 19 anos $(91,0 \%)$, residia na capital $(78,0 \%)$, vivia com o pai de seu filho $(64,5 \%)$, tinha baixa escolaridade $(55,5 \%)$, considerava-se parda $(61,5 \%)$ e dona-de-casa (53\%). Quanto aos métodos anticonceptivos, 73,5\% usaram algum método antes de engravidar, 65,5\% desejaram a gravidez e 32,5\% planejaram a gravidez. Durante a gravidez, 18,5\% tiveram internação hospitalar e 82,5\% não utilizavam drogas. Torna-se relevante a função do enfermeiro como educador em saúde para orientá-las e torná-las autônomas na promoção de sua saúde sexual.

Palavras-chave: Gravidez na adolescência. Saúde do adolescente. Enfermagem

\begin{abstract}
This study aimed to characterize the profile of puerperal adolescents in a maternity ward in Fortaleza-CE. Descriptive, transversal and quantitative study held at the Rooming Maternity School Assis Chateaubriand, in the period from March to July 2009, with 200 adolescents. The data were obtained through interviews. The majority was between 15 and 19 years old $(91.0 \%)$ resided in the capital $(78.0 \%)$ lived with the father of her child $(64.5 \%)$ had low education $(55.5 \%)$, regarded themselves as dark skin women $(61.5 \%)$ and stay-at-home (53\%). As for contraception, $73.5 \%$ used some method before becoming pregnant, $65.5 \%$ desired pregnancy and $32.5 \%$ planned pregnancy. During pregnancy, $18.5 \%$ were hospitalized and $82.5 \%$ did not use drugs. Turns relevant the role of nurses as health educators to educate them and make them autonomous in promoting sexual health.
\end{abstract}

Keywords: Pregnancy in Adolescence. Adolescent Health. Nursing.

\section{Resumen}

El objetivo de ese estudio fue caracterizar el perfil de las madres adolescentes atendidas en una maternidad de referencia en Fortaleza, Ceará. Estudio descriptivo, transversal y cuantitativo, llevado a cabo en el alojamiento conjunto de la Maternidade Escola Assis Chateaubriand, de marzo a julio de 2009, con 200 adolescentes. Los datos fueron obtenidos por medio de entrevistas. La mayoría tenía entre 15 y 19 años $(91,0 \%)$, residía en la capital (78,0\%), vivía con el padre de su hijo (64,5\%), tenía bajo nivel de educación (55,5\%), consideraba a sí misma como mixta $(61,5 \%)$ y era ama de casa $(53 \%)$. Sobre los métodos anticonceptivos, el 73,5\% utilizaron algún método antes del embarazo, el $65,5 \%$ deseaban embarazar y el $32,5 \%$ planearon el embarazo. Durante este periodo, el 18,5\% fueron hospitalizados y el $82,5 \%$ no consumía drogas. Es importante el papel de la enfermera como educadora de salud para guiarlos y hacerles autónomas en la promoción de la salud sexual.

Palabras clave: Embarazo en Adolescencia. Salud del Adolescente. Enfermería.

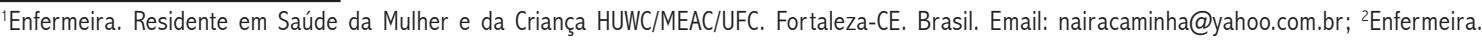
Mestranda em Enfermagem pela Universidade Federal do Ceará. Fortaleza-CE. Brasil. Email: milinha_ita@yahoo.com.br; ${ }^{3} \mathrm{Acadêmica} \mathrm{de} \mathrm{Enfermagem.}$ Bolsista do Programa de Educação Tutorial (PET) - Universidade Federal do Ceará. Fortaleza-CE. Brasil. Email: rafegobr@yahoo.com.br; ${ }^{4}$ Acadêmica de Enfermagem. Bolsista do Programa de Educação Tutorial (PET) - Universidade Federal do Ceará. Fortaleza-CE. Brasil. Email: deisemnascimento@yahoo.com.br; ${ }^{5}$ Enfermeira. Doutoranda em Enfermagem pela Universidade Federal do Ceará. Fortaleza-CE. Brasil. Email: lydia_v_freitas@yahoo.com.br; ${ }^{6}$ Enfermeira. Doutora em Enfermagem. Especialista em Enfermagem Obstétrica e Saúde da Família. Professora Adjunta Universidade Federal do Ceará. Fortaleza-CE. Brasil. E-mail: anakelve@hotmail.com.
} 


\section{INTRODUÇÃO}

A adolescência é um período caracterizado por diversas mudanças físicas e emocionais, permeado por momentos de conflitos e crises ${ }^{1}$. Deve-se considerar o adolescente como um ser humano que está sofrendo, além do que passam por mudanças corporais, mudanças no ciclo existencial, necessitando vivenciar, inovar, aventurar-se em novas experiências sem, muitas vezes, notar o quão onerosas são as responsabilidades².

De acordo com a Organização Mundial de Saúde (OMS), a adolescência compreende o período entre 10 e 19 anos; pode-se considerar a subdivisão em adolescentes menores (faixa etária entre 10 e 14 anos) e adolescentes maiores (entre 15 e 19 anos) $)^{2}$.

Nesse período torna-se notória a transição entre a infância e a vida adulta através das mudanças físicas, principalmente nas mulheres. Entre elas, pode-se citar: alargamento dos quadris e maior deposição de gordura, aparecimento de pelos pubianos e axilares, desenvolvimento mamário, menarca e início dos ciclos ovulatórios ${ }^{1}$. Como consequências principais às mudanças corporais e psicológicas durante esse período, destacam-se a maturação física e a percepção do início da potencialidade de procriação, ressaltando-se a fecundidade 3 .

Em relação à taxa de fecundidade entre mulheres adolescentes, percebe-se um aumento de $26 \%$ desde os anos 90, o que difere da taxa de fecundidade entre as demais mulheres. Esta sofreu um decréscimo nas últimas quatro décadas, sendo, em 1940, a média nacional de 6,2 filhos e, em 2000, 2,3 filhos ${ }^{4}$.

Quando são analisados os possíveis fatores etiológicos ligados ao aumento das gestações nessa faixa etária, percebese uma complexidade de fatores multicausais que tornam as adolescentes especialmente vulneráveis. No Brasil, o índice de gravidez na adolescência representa um problema social e de saúde pública, devido às repercussões biológicas, psicológicas e sociais que podem acarretar ${ }^{5}$.

Verifica-se que a vida sexual dos adolescentes é uma realidade inegável, o que torna imprescindível sua conscientização e orientação, a fim de serem evitadas gravidezes não planejadas, e problemas futuros de caráter biológico, emocional e social ${ }^{1}$.

Considerando a magnitude desta problemática, observa-se a necessidade de uma atenção pré-natal e puerperal de qualidade, holística e humanizada, em virtude das repercussões causadas à saúde materna e neonatal.

Em relação à saúde materna, as adolescentes com menos de 14 anos têm probabilidade de morrer durante a gravidez de cinco a sete vezes maior do que as outras mulheres. Já em relação à saúde neonatal, observa-se um grande número de nascimentos de crianças prematuras e/ou com peso inferior a $2500 g^{6}$.
A partir desse contexto, 0 aumento da incidência de gravidez na adolescência pode estar associado a alguns fatores, entre eles: não adoção de métodos contraceptivos ou o uso incorreto destes e o desconhecimento da fisiologia reprodutiva. Tais fatores compõem informações primordiais no contexto da atenção básica, principalmente durante as consultas de pré-natal e planejamento familiar, preconizadas pelo Ministério da Saúde7.

Dessaforma, o enfermeiro tem papelfundamental nas ações de educação junto à família, escola e comunidade, tornando o adolescente ciente de seus atos sexuais e possíveis consequências, além de promover asuaSaúdeSexual e Reprodutiva, evitando possíveis complicações que recairão não somente sobre os adolescentes, especialmente as mulheres, mas também em toda a sociedade.

Em face ao exposto, objetivou-se caracterizar o perfil das puérperas adolescentes atendidas em uma maternidade de referência de Fortaleza - Ceará.

\section{METODOLOGIA}

Trata-se de um estudo de corte transversal, descritivo e com abordagem quantitativa realizado no Alojamento Conjunto - AC da Maternidade Escola Assis Chateaubriand - MEAC, a qual presta assistência ao ciclo gravídico-puerperal, incluindo um atendimento direcionado à população adolescente, considerando as peculiaridades desse grupo populacional.

A população do estudo constituiu-se de adolescentes enquadradas na faixa etária definida pela OMS, que se estende dos 10 aos 19 anos, atendidas na instituição em estudo para resolução da gestação, a qual foi estimada a partir da quantidade de puérperas adolescentes atendidas na instituição nos últimos quatro meses anteriores à coleta de dados, sendo o N igual a 396 adolescentes. Para o cálculo amostral, utilizou-se a fórmula para cálculo de populações finitas, buscando uma amostra representativa para a maternidade estudada, adotando-se o coeficiente de confiança de $95 \%$, prevalência de $50 \%$ e erro amostral máximo permitido de $5 \%$, a partir da qual se obteve $n$ igual a aproximadamente 196 , arredondado para 200 puérperas adolescentes.

Como critério de inclusão consideraram-se as adolescentes internadas nafase de puerpério por ocasião da resolução da gravidez no AC da MEAC, no período de março a julho de 2009, e acompanhadas de seus respectivos responsáveis legais, quando menores de 18 anos, para a assinatura do Termo de Consentimento Livre e Esclarecido (TCLE). Foram excluídas do estudo as adolescentes internadas devido a abortamento ou feto natimorto.

Os dados foram coletados por meio de um formulário estruturado, elaborado pela pesquisadora, o qual continha questões fechadas e abertas, contemplando informações socioeconômicas, obstétricas e sobre os sinais e sintomas presentes na gravidez das adolescentes, sendo previamente testado, confirmando sua adequabilidade.

A coleta dos dados foi realizada, durante o puerpério imediato, por graduandos do curso de Enfermagem da Universidade Federal do Ceará - UFC, os quais receberam um treinamento prévio para uniformizar a coleta. As entrevistas 


\section{Perfil de puérperas adolescentes}

tinham início com a apresentação e assinatura do TCLE, permitindo um contato inicial com a puérpera. Durante a entrevista, preenchiase o formulário, e, ao término, realizava-se a investigação de informações necessárias no prontuário e no cartão de pré-natal da adolescente de forma a complementar as informações buscadas.

Os dados foram analisados por meio do programa Statistical Package for the Social Sciences-SPSS versão 17.0, utilizando-se a estatística simples, com frequências absolutas e relativas e o Teste Qui-quadrado, considerando correlações estatisticamente significativas as que obtiveram $p<0,05$. Foram

apresentados em tabelas e discutidos de acordo com aspectos da literatura pertinente.

Foram considerados os aspectos éticos da pesquisa envolvendo seres humanos, de acordo com o preconizado pela Resolução 196/968 , ressaltando-se a assinatura do Termo de Consentimento Livre Esclarecido por todos os responsáveis legais pelas participantes do estudo. 0 mesmo foi avaliado e aprovado pelo Comitê de Ética e Pesquisa - CEP da MEAC, mediante parecer № $76 / 08$.

\section{RESULTADOS E DISCUSSÃO}

Tabela 1-Características socioeconômicas das puérperas adolescentes atendidas na MEAC no período de março a julho. Fortaleza, Ceará, Brasil, 2009.

\begin{tabular}{lcc}
\hline Variáveis (n=200) & $\mathbf{N}$ & $\mathbf{\%}$ \\
\hline Idade & 18 & 9,0 \\
$\quad 12$ a 14 anos & 182 & 91,0 \\
$\quad 15$ a 19 anos & 156 & 78,0 \\
Procedência & 44 & 22,0 \\
$\quad$ Capital & & \\
$\quad$ Interior & 9 & 4,5 \\
Estado civil & 71 & 35,5 \\
$\quad$ Casada & 120 & 60,0 \\
$\quad$ Solteira & & \\
$\quad$ União consensual & 111 & 55,5 \\
Escolaridade & & \\
$\quad$ Fundamental incompleto (<8 anos de & 20 & 10,0 \\
estudo) & 56 & 28,0 \\
$\quad$ Fundamental completo & 13 & 6,5 \\
$\quad$ Médio incompleto & & \\
$\quad$ Médio completo & 106 & 53,0 \\
Ocupação & 73 & 36,5 \\
$\quad$ Do Lar & 9 & 4,5 \\
$\quad$ Estudante & 12 & 6,0 \\
$\quad$ Diarista / Doméstica & *Outros
\end{tabular}

*Outros: Agricultora $n=3$, Costureira $n=3$, Vendedora $n=2$, Professora $n=1$, Feirante $\mathrm{n}=1$, Escovista $\mathrm{n}=1$, Manicure $\mathrm{n}=1$

De acordo com a Tabela 1, observou-se, quanto às variáveis socioeconômicas das puérperas adolescentes, que a faixa etária predominante encontrou-se entre 15 e 19 anos, com 182 (91,0\%) jovens. A idade mais frequentemente encontrada foi 17 anos, com média de 16,76 anos e desvio-padrão de 1,598, fato que corrobora estudo realizado no município do Rio de Janeiro, em um Hospital Universitário, no qual se verificou que, entre gestantes, $86,6 \%$ eram adolescentes tardias e 11,6\% tinham entre 13 e 14 anos $^{7}$.

Vale ressaltar que $18(9,0 \%)$ puérperas tinham menos de 15 anos e 26 (13\%) adolescentes que estavam com 15 anos no pósparto imediato provavelmente iniciaram a gestação ainda com 14 anos, totalizando 44 (22\%) adolescentes que iniciaram a gestação na faixa etária de maior risco. Logo, percebe-se que um número considerável de adolescentes engravidou na faixa etária de risco, ou seja, menores de 15 anos, algo preocupante já que entre as adolescentes precoces existe uma tendência a maiores índices de prematuridade e baixo peso, quando comparadas às adolescentes mais velhas, apesar de tais diferenças não serem estatisticamente significativas?.

As participantes da pesquisa foram predominantemente procedentes da capital cearense, sendo representadas por 156 (78\%) jovens da amostra, e, dentre as 44 (22\%) restantes que residem no interior do Estado, muitas habitam a região metropolitana de
Fortaleza. A quantidade de gestantes procedentes do interior do Ceará em busca de atendimento na maternidade onde foi realizado o estudo pode ser justificada pelo fato de esta ser uma referência no Estado para gestação de risco e contar com atendimento pré-natal especializado para a população adolescente ou também por possível carência do atendimento hospitalar nos serviços de saúde do interior.

Ao analisar o estado civil, observou-se que $120(60,0 \%)$ viviam com o pai do recém-nascido ou outro companheiro, em união consensual, e somente $9(4,5 \%)$ eram casadas. Corroborando o presente estudo, observou-se que, entre grávidas adolescentes e adultas atendidas em maternidade no último trimestre da gestação em Portugal, 9,1\% e 93,8\%, respectivamente, eram casadas, já as que se apresentaram solteiras ou em regime de coabitação eram, consecutivamente, $90,9 \%$ das adolescentes e $6,2 \%$ das adultas ${ }^{10}$, sendo notória a menor frequência de casamento civil entre as adolescentes.

Quanto à escolaridade, 111 (55,5\%) adolescentes tiveram menos de oito anos de estudo, ou seja, não haviam completado o ensino fundamental, as $89(44,5 \%)$ restantes tinham oito ou mais anos de estudo; no entanto, nenhuma chegou a iniciar o ensino superior, estando isso for temente relacionado a pouca idade das participantes.

Em estudo semelhante realizado na mesma maternidade 
onde se desenvolveu a presente pesquisa, evidenciou-se que, dentre 24 adolescentes que se encontravam no último trimestre gestacional acompanhadas no serviço de pré-natal em novembro de 2006, 50,0\% tinham menos de 8 anos de estudo, as demais cursavam o ensino médio e apenas uma havia ingressado no ensino superior ${ }^{11}$. Independente do desejo de continuar ou não os estudos, esses dados revelam o prejuízo de uma gravidez precoce, principalmente ao considerar a probabilidade de essas genitoras disporem do seu tempo para cuidar dos filhos enquanto 0 companheiro provê o sustento do lar, ingressando no mercado de trabalho não especializado e mal remunerado, reproduzindo o ciclo de pobreza. Essas probabilidades podem ser embasadas por um estudo realizado em um ambulatório de uma unidade municipal de saúde em Fortaleza/CE com 40 adolescentes grávidas, o qual revelou que as três principais aspirações destas jovens são, em ordem decrescente: ser dona de casa; não ter planos; ter uma profissão e trabalhar. Entre as últimas expectativas das jovens estavam ter mais filhos e estudar ${ }^{3}$.

Considerando a variável cor, a maioria declarou-se parda, correspondendo a $123(61,5 \%)$ adolescentes, seguida por branca com $43(21,5 \%)$ e, finalmente, negra com 34 (17\%) entrevistadas.
Em pesquisa exploratório-descritiva realizada no interior do Ceará com 216 grávidas adolescentes em assistência pré-natal assistidas pela Estratégia de Saúde da Família, identificou-se uma maiorfrequência de pardas, com uma proporção de $52,3 \%$, seguida também por brancas e negras, com, respectivamente, $32,8 \% \mathrm{e}$ $10,2 \%$ dos dados válidos ${ }^{12}$.

Em relação à ocupação, 106 (53,0\%) dedararam ser donasde-casa, seguidas por estudantes, com 73 (36,5\%) entrevistadas, e 9 $(4,5 \%)$ disseram ser diaristas ou domésticas, demonstrando um dos riscos sociais da gravidez entre adolescentes, retirando da jovem as possibilidades de ascensão, pois muitas passam a ter ocupação de dona-de-casa, abandonandoestudoseempregos, ou quandoempregadas, ocupam cargos profissionais que proporcionam baixa renda.

Divergindo do presente estudo, observou-se, em estudo realizado com adolescentes no Centro de Desenvolvimento Familiar - CEDEFAM, instituição pública vinculada à Universidade Federal do Ceará, que 45,0\%, tinham como ocupação estudar, seguida por atividades do lar com $32,5 \%$ e trabalho remunerado com $22,5 \%{ }^{13}$. Considerando que as adolescentes do citado estudo estavam grávidas e nosso estudo tratou de puérperas, tal diferença pode estar relacionada a estas adolescentes abandonarem o estudo próximo ao final ou após o desfecho da gestação.

Tabela 2 - Caracterização da gestação atual das puérperas adolescentes atendidas na MEAC no período de março a julho. Fortaleza, Ceará, Brasil, 2009.

\begin{tabular}{|c|c|c|}
\hline Variáveis $(n=200)$ & $\mathbf{N}$ & $\%$ \\
\hline \multicolumn{3}{|l|}{$\begin{array}{l}\text { Engravidou do primeiro } \\
\text { parceiro }\end{array}$} \\
\hline Sim & 112 & 56,0 \\
\hline Não & 88 & 44,0 \\
\hline \multicolumn{3}{|l|}{ Utilizou método contraceptivo } \\
\hline Sim & 147 & 73,5 \\
\hline Não & 53 & 26,5 \\
\hline \multicolumn{3}{|l|}{ Gravidez planejada } \\
\hline Sim & 65 & 32,5 \\
\hline Não & 135 & 67,5 \\
\hline \multicolumn{3}{|l|}{ Gravidez desejada } \\
\hline Sim & 135 & 67,5 \\
\hline Não & 65 & 32,5 \\
\hline \multicolumn{3}{|l|}{ Internações na gestaç̃o atual } \\
\hline Não & 163 & 81,5 \\
\hline Sim & 37 & 18,5 \\
\hline \multicolumn{3}{|l|}{ Motivos de internação } \\
\hline Sangramento transvaginal & 11 & 5,5 \\
\hline Pré-eclâmpsia / eclâmpsia & 7 & 3,5 \\
\hline Infecção do trato urinário & 7 & 3,5 \\
\hline Amniorrexe & 4 & 2,0 \\
\hline Trabalho de parto prematuro & 4 & 2,0 \\
\hline Alterações gastrointestinais & 1 & 0,5 \\
\hline Hipoglicemia & 1 & 0,5 \\
\hline Dor em baixo ventre & 2 & 1,0 \\
\hline Não se aplica & 163 & 81,5 \\
\hline \multicolumn{3}{|l|}{ Hábitos } \\
\hline Fumo & 12 & 6,0 \\
\hline Álcool & 10 & 5,0 \\
\hline Fumo e álcool & 9 & 4,5 \\
\hline Fumo e drogas & 1 & 0,5 \\
\hline Fumo, álcool e drogas & 3 & 1,5 \\
\hline Nenhum & 165 & 82,5 \\
\hline \multicolumn{3}{|l|}{ Descoberta da gravidez } \\
\hline BHCG & 96 & 48,0 \\
\hline Exame de farmácia & 30 & 15,0 \\
\hline Sintomatologia & 58 & 29,0 \\
\hline TIG & 15 & 7,5 \\
\hline Não respondeu & 1 & 0,5 \\
\hline
\end{tabular}




\section{Perfil de puérperas adolescentes}

A partir da Tabela 2, quanto à história da gestação atual, ao serem questionadas sobre ter ou não engravidado do primeiro parceiro, $112(56 \%)$ adolescentes afirmaram que sim, demonstrando que as relações sexuais podem ter ocorrido de forma intempestiva ou sem a utilização de métodos anticonceptivos eficazes, resultando, assim, em gravidez não planejada.

Com relação aos métodos anticonceptivos, percebese uma parcela importante de adolescentes $(26,5 \%)$ que não aderiram ao uso de anticonceptivos, seja pelo plano e/ ou desejo de engravidar, seja pela dificuldade de acesso à informação e ao método anticonceptivo propriamente dito.

Em estudo realizado em uma unidade de saúde da família de Boa Esperança/MG, 18,2\% das adolescentes grávidas entrevistadas referiram dificuldade de acesso aos métodos anticonceptivos, todas conheciam o condom e $86,4 \%$ conheciam os anticoncepcionais orais. No entanto, somente $31,8 \%$ delas referiram usar algum método. Este índice tão inferior ao encontrado no presente estudo pode estar relacionado ao fato da referida unidade de saúde ter índice de gravidez entre adolescentes maior que a média nacional. Corroborando a pesquisa, identificou-se o condom e a pílula entre os métodos mais frequentemente utilizados pelas jovens ${ }^{14}$.

Quanto ao planejamento da gravidez, a maioria, ou seja, 135 adolescentes $(67,5 \%)$ relataram não tê-la planejado. Este fato também foi encontrado em estudo realizado em um Hospital Universitário no Rio de Janeiro com 112 adolescentes grávidas, o qual constatou que, entre as entrevistadas, 84 (75\%) afirmaram que não haviam planejado a gravidez ${ }^{7}$.

Em contrapartida, no presente estudo, um grande número de adolescentes, 135 (67,5\%) afirmou que a gravidez foi desejada, apesar de não planejada. Corroborando outro estudo realizado no Hospital Universitário do município do Rio de Janeiro com gestantes adolescentes, o qual demonstrou que, apesar da gravidez não ter sido planejada e de ter sido um evento inesperado na vida das jovens, 66 adolescentes, ao descobrirem que estavam grávidas, tiveram sentimentos positivos diante da notícia ${ }^{7}$.

Verificou-se significância estatística entre o estado civil das jovens e o planejamento da gravidez $\left(x^{2}=17,017 ; p<0,001\right)$, podendo-se inferir que as adolescentes que tinham companheiros (casadas ou unidas consensualmente) apresentaram tendência maior ao planejamento da gestação. Encontrou-se ainda significância estatística entre o estado civil e o desejo de engravidar ( $\left.x^{2}=7,929 ; p=0,005\right)$, de forma que as mulheres que vivem com seus companheiros apresentaram uma tendência maior de desejar uma gestação. Esse resultado já era esperado, considerando que a gravidez é mais aceita pela sociedade quando ocorre em uma relação matrimonial ou união estável. No entanto, no presente estudo não é possível determinar qual fenômeno ocorreu primeiro, se a união ou a gravidez, visto que tal variável não foi investigada no instrumento de coleta de dados.

Em relação ao internamento durante a gestação, 37 puérperas $(18,5 \%)$ relataram internamento. Entre as principais causas de hospitalização, encontraram-se sangramento transvaginal, síndrome hipertensiva gestacional e infecções do trato urinário.

Em estudo semelhante realizado com 84 adolescentes moradoras de São José do Rio Preto que tiveram filhos no período de 01 de outubro a 01 de dezembro de 2004, pelo Sistema Único de Saúde (SUS), verificaramse entre as principais intercorrências clínicas: infecção urinária $(35,7 \%)$, sangramento vaginal $(14,3 \%)$, pressão arterial elevada (14,2\%), diabetes gestacional $(2,4 \%)$ e eclampsia (1,2\%). E 35,7\% das adolescentes necessitaram de internação durante a gravidez por causa destas intercorrências ${ }^{15}$.

Constatou-se significância estatística entre a idade e a necessidade de internações durante a gestação atual $\left(x^{2}=13,017 ; p=0,01\right)$, demonstrando que quanto menor a idade (12-14 anos) maior a probabilidade de haver a necessidade de internação, fato esperado visto tratar-se da faixa etária de maior risco de complicações durante a adolescência. Tais dados indicam que as complicações na gestação em adolescentes são muito frequentes, ressaltando-se a importância de um acompanhamento prénatal direcionado para essa faixa etária.

Quanto à utilização de drogas durante a gestação, observou-se que grande parte da amostra, ou seja 165 mulheres (82,5\%), não utilizou nenhum tipo de droga. Contudo, destaca-se que $3(1,5 \%)$ tinham como hábito 0 uso simultâneo de drogas ilícitas, álcool e cigarros, sendo também visível que o uso de drogas ilícitas apresentouse sempre associado ao tabagismo ou também ao etilismo.

Verificou-se em uma maternidade do município de São Paulo, entre 1.000 adolescentes grávidas internadas para parturição ou curetagem pósabortamento, o uso de tabaco, álcool e drogas ilícitas entre 17,3\%, 26,3\% e 1,7\% das jovens. ${ }^{16}$ Tais frequências são preocupantes, evidenciando a maior necessidade de orientação durante a assistência pré-natal sobre os malefícios do uso de drogas para a gestante e o feto.

Em relação à descoberta da gestação, 96 (48\%) relataram que foi por meio do exame de sangue bHCG, 58 (29\%) foram apenas pela sintomatologia e $30(15,0 \%)$, pelo exame de farmácia. Isso pode sugerir que as adolescentes estão buscando mais os serviços de saúde ao suspeitarem de uma gravidez. 
Tabela 3 - Distribuição dos sinais e sintomas vivenciados durante a gestação por puérperas adolescentes atendidas na MEAC no período de março a julho. Fortaleza, Ceará, Brasil, 2009.

\begin{tabular}{|ccc|}
\hline Variáveis (n=200) & $\mathbf{N}$ & $\mathbf{\%}$ \\
\hline Nictúria & 188 & 94,0 \\
\hline Náuseas, vômitos e & 133 & 66,5 \\
tonturas & & \\
$\quad$ Lombalgia & 111 & 55,5 \\
\hline Cãibras & 109 & 54,5 \\
\hline Pirose & 103 & 51,5 \\
\hline Cefaleia & 96 & 48,0 \\
\hline Estrias & 96 & 48,0 \\
\hline Dispneia & 74 & 37,0 \\
\hline Corrimento vaginal & 67 & 33,5 \\
\hline Cólicas & 66 & 33,0 \\
\hline Fraquezas e/ou desmaios & 54 & 27,0 \\
\hline Sialorreia & 45 & 22,5 \\
\hline Flatulência & 45 & 22,5 \\
\hline Constipação intestinal & 41 & 20,5 \\
\hline Dor nas mamas & 38 & 19,0 \\
\hline Gengivite & 26 & 13,0 \\
\hline Cloasma gravídico & 21 & 10,5 \\
\hline Varizes & 19 & 9,5 \\
\hline Hemorroidas & 4 & 2,0 \\
\hline
\end{tabular}

$\mathrm{Na}$ Tabela 3, verificam-se os sinais e sintomas apresentados pelas adolescentes durante a gestação atual, sendo a nictúria o sintoma mais referido, por 188 (94\%) adolescentes, seguidos por náuseas, vômitos e tonturas ,sentidos por $133(66,5 \%)$ entrevistadas. A lombalgia foi encontrada em 111 (55,5\%) adolescentes. Já as hemorroidas podem ser consideradas o sintoma menos evidente nesta população, presente em 4 (2\%) jovens. Em estudo realizado com 11 gestantes de baixo risco em uma Maternidade Pública no interior de Goiás, identificou-se que todas apresentaram urgência urinária e nictúria devido à pressão do útero sobre a bexiga, sendo esse sintoma mais comumente encontrado no terceiro trimestre da gravidez ${ }^{17}$.

Em Massapê/CE, em um estudo realizado com puérperas adolescentes, observou-se, durante o período gestacional, uma menor frequência de náuseas, vômitos e tonturas sendo sua frequência de $25,0 \%, 20,0 \%$ e 15,0\%, respectivamente ${ }^{18}$. A diferença entre os resultados pode estar relacionada ao fato de a pesquisa destes sintomas ter ocorrido de forma conjunta em um estudo e isoladamente no outro.

Os sinais e sintomas da gestação estão diretamente associados ao estilo de vida da gestante. Cabe ao enfermeiro orientar as gestantes durante as consultas de pré-natal para que elas obtenham instruções corretas acerca da manutenção de hábitos saudáveis, como a ingestão de alimentos adequados e a prática de exercícios físicos direcionados à gestante, apresentando os meios para se ter uma melhor qualidade de vida e obter alívio destes sintomas. Além disso, a gestante deve ser orientada sobre as transformações de seu corpo durante este período e fazer uma estimativa das possíveis repercussões que isso trará ao seu bem-estar para que encontre meios de adaptar-se eles.

\section{CONCLUSÕES}

Concluí-se que a gravidez na adolescência é um fenômeno que continua ocorrendo com frequência em nosso país, visto que a taxa de fecundidade nesta população vem crescendo ao longo dos anos, principalmente entre as jovens com idade inferior a 20 anos.

Pode-se inferir que o perfil das adolescentes grávidas do estudo são mulheres de 15 a 19 anos, que têm baixa escolaridade e que estão unidas consensualmente ao parceiro.

Vale destacar uma sensível adesão das adolescentes aos métodos anticonceptivos antes de vivenciarem a gestação. No entanto, pode-se inferir o provável uso inadequado dos métodos. Evidenciou-se também o desejo de engravidar entre as jovens; mesmo não tendo planejado uma gravidez, consideraram-na bem-vinda, o que indica aceitação do processo gestacional.

Em relação aos riscos envolvidos em uma gravidez precoce, percebeu-se que a maioria não necessitou de internação hospitalar durante o processo gestacional, apesar de vivenciarem os sinais e sintomas comuns à gestação.

Diante do exposto, verifica-se o elevado número de gravidez na adolescência, demonstrando a necessidade de orientações direcionadas para a população jovem, em linguagem clara e acessível, acerca do processo reprodutivo e do planejamento familiar, destacando-se os tipos de métodos contraceptivos e seu uso de forma adequada, possibilitando um maior conhecimento e empoderamento quanto à tomada de decisão na adoção de um comportamento sexual saudável.

Destaca-se a importância do enfermeiro como um verdadeiro educador em saúde e sua inserção no mundo escolar com o objetivo de realizar um acompanhamento dos 
adolescentes, utilizando variadas estratégias de educação em saúde que visem capacitá-los e torná-los autônomos na sua promoção da saúde, evitando, dessa forma, os principais problemas que atingem essa faixa etária, como a gravidez não planejada e as doenças sexualmente transmissíveis.

\section{REFERÊNCIAS}

1. Beretta MIR, Freitas MA, Dupas G, Fabbro MRC, Ruggiero EMS. A construção de um projeto na maternidade adolescente: relato de experiência. Rev Esc Enferm USP. 2011; 45(2): 533-36.

2.World Health Organization-WHO. Adolescent Hearth, 2008. Disponível em: http://www.who.int/topics/adolescent health/en/.

3. Arcanjo CM, Oliveira MIV, Bezerra G. Gravidez em adolescentes de uma unidade municipal de saúde em Fortaleza, Ceará. Esc. Anna Nery. 2007; 11(3): 445-51.

4. Ministério da Saúde(BR). Databases. Indicadores e dados básicosIDB. Brasil 2008. [citado 2009 dez 11]. Disponível em: http:// tabnet.datasus.gov.br/cgi/idb2008/matriz.htm.

5. Carvacho IE, Silva JLP, Mello MB. Conhecimento de adolescentes grávidas sobre anatomia e fisiologia da reprodução. Rev Assoc Med Bras. 2008; 54(1): 29-35.

6. Melo MCP, Coelho EAC. Integralidade e cuidado a grávidas adolescentes na Atenção Básica. Cienc Saude Colet. 2011; 16(5): 2549-58.

7. Spindola T, Silva LFF. Perfil epidemiológico de adolescentes atendidas no pré-natal de um hospital universitário. Esc. Anna Nery. 2009 mar; 13(1): 99-107.

8. Ministério da Saúde(BR). Conselho Nacional de Saúde. Resolução n ${ }^{0}$ 196, de 10 de outubro de 1996. Diretrizes e normas regulamentadoras de pesquisas envolvendo seres humanos. Bioética, Brasília, 1996; 4(2 supl): 15-25.

9. Goldenberg P, Figueiredo MCT, Silva RS. Gravidez na adolescência, prénatal e resultados perinatais em Montes Claros, Minas Gerais, Brasil. Cad Saude Publica. 2005 ago; 21(4).

10. Figueiredo B, Pacheco A, Margarinho R. Grávidas adolescentes e grávidas adultas: diferentes circunstâncias de risco? Acta Med Port. 2005 mar/abr;18(2): 97-105.

11. Oliveira EMA, Moura ERF, Pinheiro PNC, Eduardo KGT. Histórico contraceptivo de adolescentes grávidas e seus sentimentos quando a gravidez e ao futuro profissional. Rev Eletr Enferm. [on- line] 2008 [citado 2010 jun 05]; 10(2): 484-90. Disponível em: http://www.fen.ufg.br/ revista/v10/n2/v10n2a19.htm.

12. Ximenes Neto FRG, Dias MSA, Rocha J, Cunha ICKO. Gravidez na adolescência: motivos e percepções de adolescentes. Rev Bras Enferm. 2007 maio/jun; 60(3): 279-85.
13. Belarmino GO, Moura ERF, Oliveira NC, Freitas GL. Risco nutricional entre gestantes adolescentes. Acta Paul Enferm. 2009; 22(2):169-75.

14. Gradim C, Ferreira M, Moraes M. 0 perfil das grávidas adolescentes em uma unidade de saúde da família de minas gerais. Rev APS. [on-line] 2010 mar [citado 2010 jun 05]; 13(1): 55-61.Disponível em: < http:// ojs.hurevista.ufff.br/index.php/aps/article/view/505/297>.

15. Faria DGS, Zanetta DMT. Perfil de mães adolescentes de São José do Rio Preto, Brasil e cuidados na assistência pré-natal. Arq Cienc Saude. 2008 mar; 15(1):17-23.

16. Chalem E, Mitsuhiro SS, Ferri CP, Barros MCM, Guinsburg R, Laranjeira R. Gravidez na adolescência: perfil sócio-demográfico e comportamental de uma população da periferia de São Paulo, Brasil. Cad Saude Publica. 2007 jan; 23(1).

17. Pereira SVM, Bachion MM. Diagnósticos de enfermagem identificados em gestantes durante o pré-natal. Rev Bras Enferm. 2005 dez; 58(6): 659-64.

18. Ximenes Neto FRG, Marques MS, Rocha J. Problemas vivenciados pelas adolescentes durante a gestação. Enferm Glob. 2008; (12):1-11. 